\title{
Blind separation of internal combustion engine vibration signals by deflation method
}

\author{
Xianhua Liu $^{\mathrm{a}^{*}}$, R. B. Randall ${ }^{\mathrm{b}}$ and Jérôme Antoni ${ }^{\mathrm{c}}$ \\ ${ }^{a}$ School of Mechanical Engineering \\ The University of Western Australia, Perth 6009, Australia \\ ${ }^{b}$ School of Mechanical and Manufacturing Engineering \\ The University of New South Wales, Sydney 2052, Australia \\ ${ }^{c}$ Laboratoire Roberval de Mécanique \\ Universitè de Technologie de Compiègne, 60205 Compiègne, France
}

\begin{abstract}
Internal combustion engines have several vibration sources, such as combustion, fuel injection, piston slap and valve operation. It is necessary to separate the different vibration sources and then analyse each of them individually. This paper attempts to separate the vibration sources by blind source separation techniques and proposes a combination of Blind Least Mean Square algorithm with a deflation method to separate several sources. The combined methods are first verified by separating simulated sources and then applied to engine signals. Separation results show that cylinder pressure consists of two sources, one is a smooth low frequency part due to compression and expansion caused by piston movement; the other is the high frequency part due to combustion.
\end{abstract}

Keywords: Blind source separation; deflation method; IC engine diagnostics; piston slap

\section{Introduction}

Internal combustion engine noise has always been a research interest in mechanical engineering. Reducing the noise means creating a better environment as well as a good quality engine in terms of improved combustion process, improved fuel injection, and reduced piston slaps etc. For this purpose there is a need to measure vibration signals of engines and analyse them. Due to the measured vibration signals are combination effects of several vibration sources. It is ideal if they can be separated and be analysed individually. However, traditional frequency analysis techniques are not very useful due to overlap of the different sources over a wide frequency range. In recent years Blind Source Separation (BSS) techniques have emerged. Because they separate or recover the system and the input sources by using only the measured system output data, they offer a promising approach to tackle signal processing problems in communication, image processing, speech acoustics etc. There is now an increasing interest in applying BSS method to mechanical engineering problems. A good example is the special issue of 15 papers on BSS in the MSSP journal [1], where we published a paper on separating piston slaps from other vibration sources [2]. The BSS method used in the previous paper can only recover one supergaussian and/or one subgaussian sources. For separating more sources it proposed to combine a deflation method. In this paper we will describe this combined method in more detail and carry out both simulated signal and practical engine signal separations. 


\section{Blind source separation problem and algorithms}

Blind Source Separation deals with the problem of finding both the unknown input sources and unknown mixing system from only observed output mixtures. This problem model has been widely described in many papers and books on blind source separation [3-4]. In mechanical vibration problems, forces acted on mechanical structures are usually the input sources; mechanical structures are the mixing systems which generate vibration responses to the input forces; and the measured vibration signals are output of the mechanical structures due to the input forces. Mechanical vibrations caused by multiple forces follow the multichannel convolved mixing model as shown in equation (1). Here $\mathbf{s}\left(t_{n}\right)$ is a vector of sources at time instant $t_{n} ; \mathbf{x}\left(t_{n}\right)$ a vector of mixtures; and $\mathbf{H}$ is a matrix of impulse responses representing the system property. Note that in this paper, matrices are denoted by capital bold letters; vectors are denoted by small bold letters; scalars are denoted by italic letters.

For multichannel convolved mixing model,

$$
\mathbf{x}\left(t_{k}\right)=\mathbf{H} * \mathbf{s}\left(t_{k}\right)
$$

Where $\mathbf{s}\left(t_{k}\right)=\left\{s_{i}\left(t_{k}\right)\right\}$

$i=1,2, \ldots, M$, source vector as input to the system

$$
\mathbf{x}\left(t_{k}\right)=\left\{x_{j}\left(t_{k}\right)\right\}
$$$$
j=1,2, \ldots, N \text {, observed signals as system output }
$$

$$
\mathbf{H}=\left[\mathbf{h}_{j i}\right]_{N \times M}
$$

Matrix of impulse responses as mixing system

$$
*
$$

\section{Convolution operator}

$\mathbf{h}_{j i}\left(t_{k}\right)=\left[\begin{array}{llll}h_{j i 0}\left(t_{k}\right) & h_{j i 1}\left(t_{k}\right) & \ldots & h_{j i(L-1)}\left(t_{k}\right)\end{array}\right]$, impulse response from source $i$ to mixture $j$

The method for recovering the unknown sources is to construct an objective or cost function and derive an adapting algorithm to optimize the function by either maximizing or minimizing it. In paper the previous paper we used the BLMS algorithm, which is based on the Central Limit Theorem and derived by Kullback-Leibler Divergence and approximated by Gray's variable norm to separate piston slaps from other vibration sources of internal combustion engines [2]. The algorithm is expressed in equation (2-6). The entry $\mathbf{x}_{j}(k)$ is the section of the last $L$ samples of the $j^{\text {th }}$ mixture component in time-reversed order as indicated in (4), and $L$ is filter length; the $\mathbf{w}_{i j}(k)$ denotes the FIR filter vector of length $L$ at the $i^{\text {th }}$ row and $j^{\text {th }}$ column of the inverse system $\mathbf{W}$ for iteration step $k$ as in (5). The ${ }^{T}$ in equation (2) denotes the transpose of the row vector $\mathbf{x}_{j}(k)$. Note that the time instant index $t_{k}$ has been simplified as sample index $\mathrm{k}$ since all the signals are used in the form of discrete data.

$$
\begin{aligned}
& \hat{s}_{i}(k)=\sum_{j=1}^{N} \mathbf{w}_{i j}(k) \mathbf{x}_{j}^{T}(k) \\
& \mathbf{w}_{i j}(k+1)=\mathbf{w}_{i j}(k)+\mu\left(\hat{s}_{i}(k)-g\left(\hat{s}_{i}(k)\right)\right) \mathbf{x}_{j}(k) \\
\text { Where } & \mathbf{x}_{j}(k)=\left[\begin{array}{llll}
x_{j}(k) & x_{j}(k-1) & \ldots & x_{j}(k-L+1)
\end{array}\right] \\
& \mathbf{w}_{i j}(k)=\left[\begin{array}{llll}
w_{i j 0}(k) & w_{i j 1}(k) & \ldots & w_{i j(L-1)}(k)
\end{array}\right] \\
& g\left(\hat{s}_{i}(k)\right)=\frac{E\left|\hat{s}_{i}\right|^{2}}{E\left|\hat{s}_{i}\right|^{\alpha}} \hat{s}_{i}(k)\left|\hat{s}_{i}(k)\right|^{\alpha-2}
\end{aligned}
$$


For supergaussian sources with high kurtosis such as piston slap, let $\alpha=1$; for subgaussian sources, let $\alpha=4$. The algorithm will drive the recovered sources as far away from a Gaussian distribution as possible. However this algorithm only finds one source for each positive or negative $\alpha$ value. This can be both an advantage and a disadvantage. The advantage is that it is not necessary to know the exact number of sources present. The disadvantage is that if the signals contain more than one source with approximately the same value of kurtosis, only one source usually the stronger one will be found.

\section{Combining deflation method with the BLMS algorithm}

For separating more sources a learning algorithm called deflation can be used to extract source responses from the mixtures using the recovered source. After deflation, the above BLMS algorithm can be applied to the remaining mixtures to separate further sources. One deflation algorithm has been proposed by Chio and Cichocki in 1998 by minimizing the energy cost function [5]. Here we briefly describe the basic theory on which this deflation algorithm is based and show how it can be combined with the BLMS algorithm to separate more sources.

For independent sources, the energy of their sum equals the sum of the energy in each source. This is expressed in equations (7-8), where $E$ denotes expectation or average and in (8) we invoke the fact that the cross correlation between two independent signals is zero. This theory can be applied to the BSS mixing model for the reason that scaled or filtered sources are still independent sources. For example if $x$ is a mixture of a number of filtered sources as in (9), then equation (10) exists.

$$
\begin{aligned}
& x=\sum_{i=1}^{M} s_{i} \\
& E x^{2}=E\left(\sum_{i=1}^{M} s_{i}\right)^{2}=\sum_{i=1}^{M} E s_{i}^{2} \\
& x=\sum_{i=1}^{M} h_{i} * s_{i} \\
& E x^{2}=E\left(\sum_{i=1}^{M} h_{i} * s_{i}\right)^{2}=\sum_{i=1}^{M} E\left(h_{i} * s_{i}\right)^{2}
\end{aligned}
$$

We have recovered one source by the BLMS algorithm and now want to recover more sources by combining a deflation method. If the first source can be recovered as in (11), we then need to deflate or subtract its contribution from the mixture $\mathbf{x}$ so that the remaining mixture contains only the contributions of other sources, which enables the second source to be recovered by the BLMS algorithm. If we denote the remaining mixture as $\mathbf{x}_{1}$ after first deflation, then it can be obtained by (12). Here $\widetilde{w}_{1 j}$ is an inverse filter for deflating the contribution of source $\hat{s}_{1}$ from mixture $x_{j}$. Under the assumption of independent sources, based on the energy superposition principle described in (7-10), there exists the minimizing energy cost function (13) proposed by Chio and Cichocki in 1998 [5]. This leads to the stochastic updating rule (14) for obtaining the deflating filter $\widetilde{w}_{1 j}$. Now equations (12-14) have constructed a deflation algorithm. By using the BLMS algorithm and the deflation algorithm alternatively more sources will be blindly recovered.

$$
\begin{aligned}
& \hat{s}_{1}(k)=\sum_{j=1}^{N} \mathbf{w}_{1 j} \mathbf{x}_{j}(k)=\sum_{j=1}^{N} \sum_{p=0}^{L-1} w_{1 j p} X_{j}(k-p) \\
& x_{1 j}(k)=x_{j}(k)-\widetilde{\mathbf{w}}_{1 j} \hat{\mathbf{s}}_{1}(k)=x_{j}(k)-\sum_{p=0}^{L-1} \widetilde{w}_{1 j p} \hat{s}_{1}(k-p)
\end{aligned}
$$




$$
\begin{aligned}
& \rho=\frac{1}{2} E\left\{\sum_{j=1}^{N} x_{1 j}(k)^{2}\right\} \\
& \widetilde{w}_{1 j p}(k+1)=\widetilde{w}_{1 j p}(k)-\mu x_{1 j}(k) \hat{s}_{1}(k-p)
\end{aligned}
$$

We verify combining the deflation method into the BLMS algorithm by carrying out some simulation separation experiment and make an attempt to separate more engine sources afterward.

\section{Simulation verification}

The purpose of separation simulation is to verify the deflation method (12-14), so as to show its validity for later mechanical application. Here we generated four sources as shown in Fig. 1, which are: a sine wave, a sparse impulsive source, a uniform distribution and a gamma distribution sources with normalised kurtosis equal to $-1.5,32.2,-1.2$ and 10.3 respectively, and with expected energy or variance equal to $0.50,0.02,0.33$ and 0.97 respectively. The length of the source signals is 10000 samples. Their multichannel convolved mixtures are shown in Fig. 2 and the mixing system is shown in Fig. 3, which is randomly generated double exponentially decaying impulse response functions of 64 samples in length and has been checked for existence of an inverse system.

Fig. 4 shows the separated sources. The $4^{\text {th }}$ source with gamma distribution has been separated as the first source. By comparing with the original source in Fig. 1, it is seen that this first separated source is recovered accurately except a delay. The initial section of zero values is due to the filter length occupied in convolution, which can be either set to be zero, or left as noise because of not being able to be recovered correctly. To avoid affecting the deflation and separation process in the following steps it has been simply set it to zero values here, which means that this section of signals will not be calculated. This will cause the mixtures and recovered sources in later steps to become shorter. However it is compensated by better separation quality for the rest of the signals.

After deflation of the first separated source, the second source recovered is the impulsive source. It is correctly recovered except with noticeable noise as shown in the second row. Note that the two supergaussian sources are recovered because we have used $\alpha=1$ in the BLMS algorithm. It is interesting to note that, of the two extracted supergaussian sources, the Gamma distribution is extracted first even though the impulsive source has the maximum kurtosis. This is because that the Gamma distribution source has larger energy or variance. It is also interesting to note that, although the spare impulsive source has very small energy, it is still recovered correctly. On the other hand, both sources offer optimum solution during the iteration process of filter coefficient adaptation. Which source is separated first can also depend on the initial condition of the iteration process, i.e. the initial values of the filter $\mathbf{w}$.

After deflating the contributions of the two supergaussian sources, only the mixture of the two subgaussian sources remains. Thus the BLMS algorithm is changed over to $\alpha=4$ for separating them. The third recovered source is the uniform source, which is in fact both smaller in terms of kurtosis and variance than the sine wave.

The sine wave signal is separated at last from the remaining noise. The noise is caused by the errors of the previous BLMS and deflating process. Errors accumulate in later separation steps and hence the separation accuracy will decrease as more sources are extracted. This is a typical character of deflation algorithms as described by Hyvärinen et al in 2001 [6]. However we still can recognize that the last separated source is a sine wave. If further signal processing is done on the recovered sine wave, a more accurate sine wave can be obtained. 
In summary, the combination of BLMS algorithms with the deflation method to separate more sources has been verified to be successful for simulated signals. Note that a supergaussian source and a subgaussian source can be extracted at the same time by adopting both $\alpha=1$ and $\alpha=4$. However we used the simpler way to recover the sources one by one.
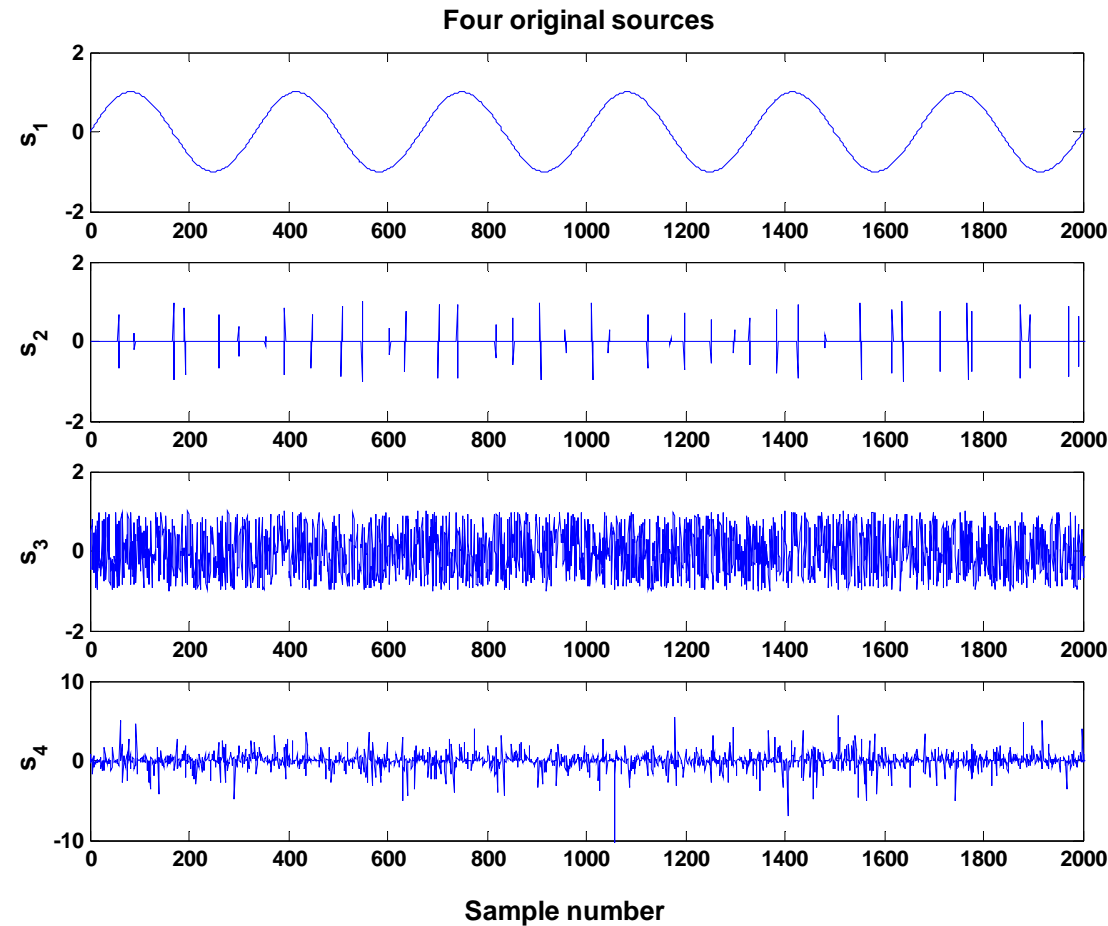

Fig. 1. Four artificially generated sources
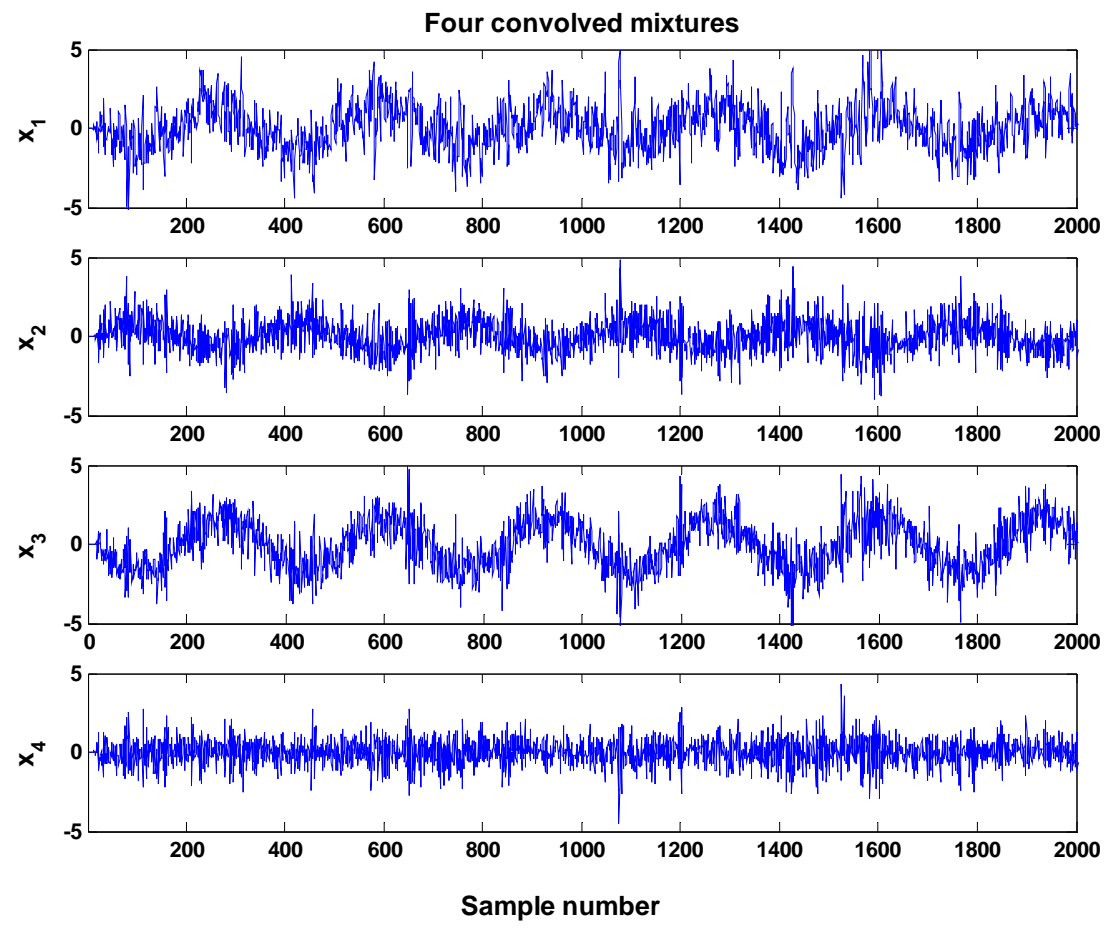

Fig. 2. Four multichannel convolved mixtures 


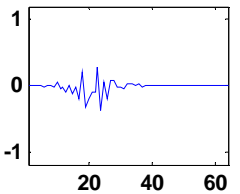

Randomly generated mixing system $\mathrm{H}=\left[\mathrm{h}_{\mathrm{ij}}\right]$
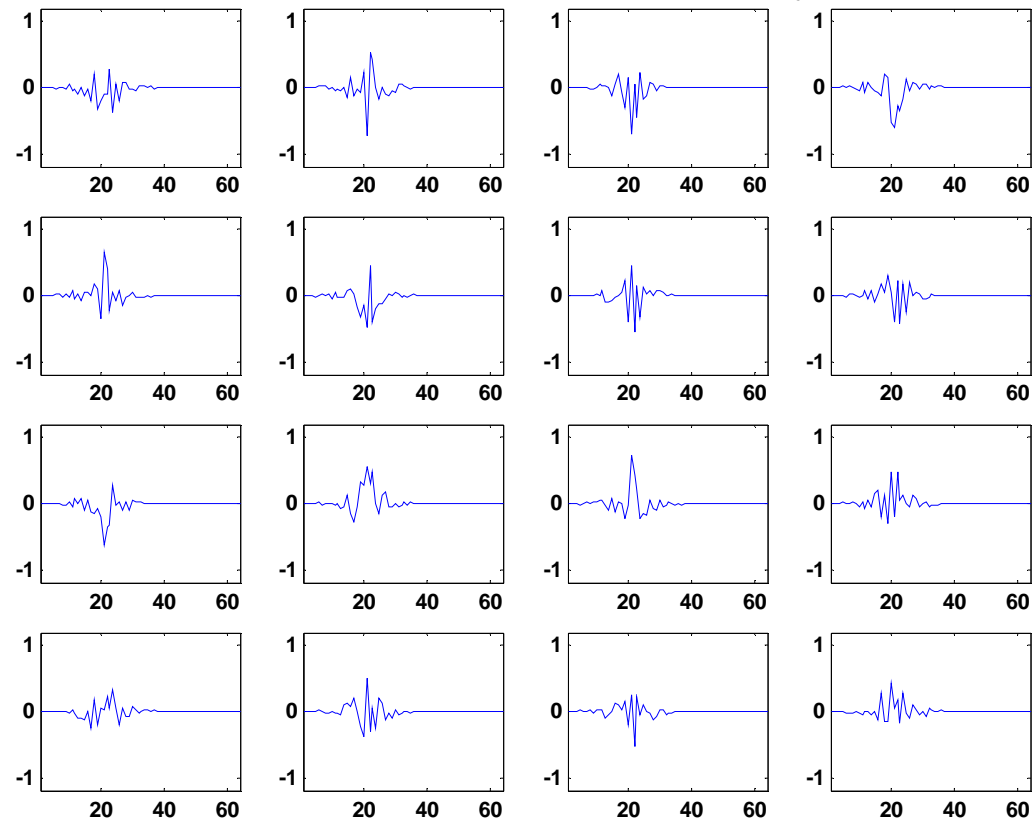

Filter sample number

Fig. 3. Sixteen randomly generated filters to form a 4 inputs 4 outputs multichannel convolutive mixing system
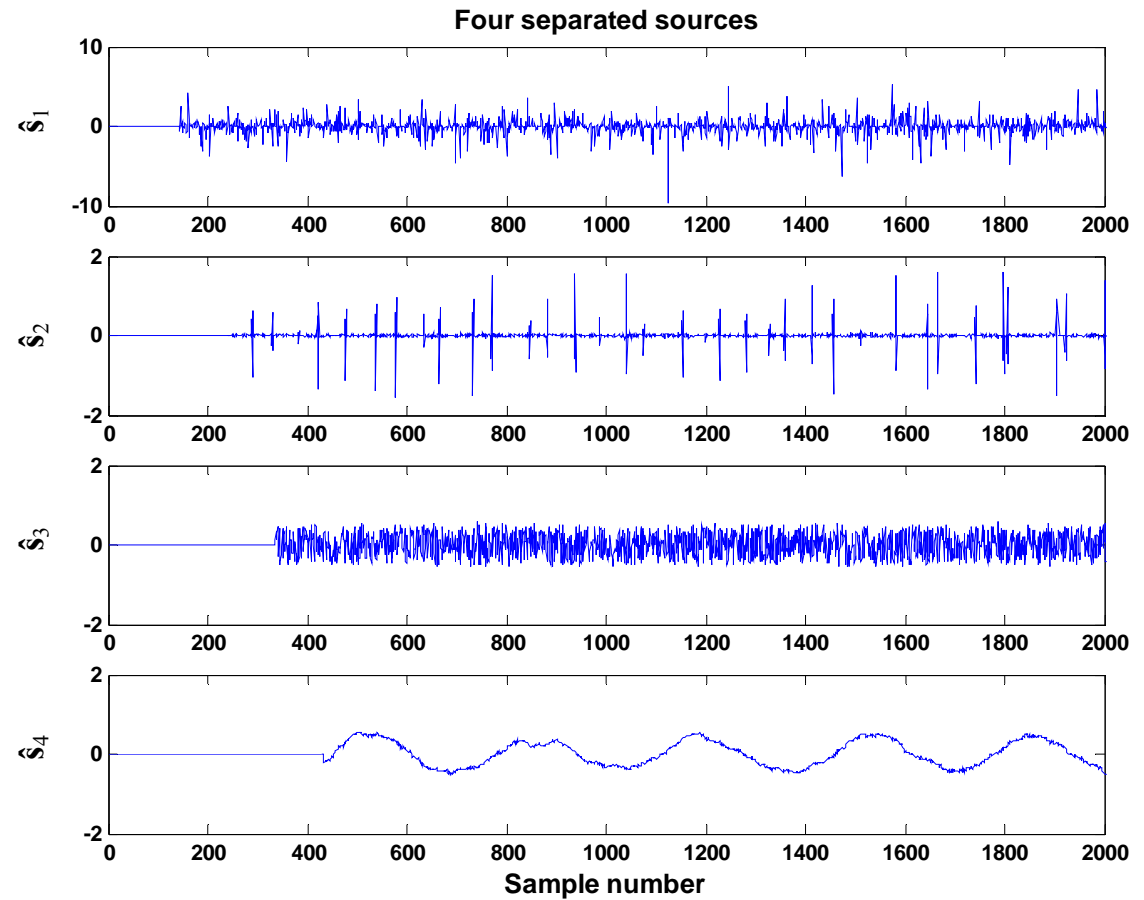

Fig. 4. Four separated sources by combining the deflation method with the BLMS algorithm 


\section{Blind separation of diesel engine vibration signals}

In this section we apply the combined method to separate internal combustion engine sources. Signals from a four-cylinder diesel engine with faulty piston were measured including vibration acceleration, cylinder pressure, fuel injection and once-per-revolution tacho of the crankshaft. Only the sections of the measured signals corresponding to combustion in one cylinder around its piston Top Dead Centre are used. During this short time range the vibration is caused mainly by the operation of this cylinder such as fuel injection, combustion, and piston slap with the rest being regarded as background noise. Because of the piston moving along the cylinder, the system is actually a time-varying system. However if only the section of the signals around TDC is being considered, for example within $-25^{\circ} \sim 45^{\circ}$ crank angle, the piston only moves slowly over a short distance and hence the system can be regarded as approximately time invariant. In the previous paper, separation results by using only the BLMS algorithm have been presented, here we present separation results of more sources by combining the deflation algorithm.

First an operating speed of $1210 \mathrm{rpm}$ of the engine is used. Fig. 5 shows the four measured vibration acceleration signals from the engine case around one cylinder of interest. Three sources are separated from four measured acceleration signals. Using the BLMS algorithm the first source $\hat{\mathbf{s}}_{1}$ is separated as the piston slap as shown in Fig. 6, which is regarded as piston slap signals as discussed in the previous paper [2]. After deflating the contribution of the recovered piston slap, the second source $\hat{\mathbf{s}}_{2}$ is separated from the remaining mixtures. This source is like a slow sine wave, which is subgaussian and is interpreted as a filtered version of the part of cylinder pressure. It is mainly the part of cylinder pressure caused by compression and expansion of fuel and gases in the cylinder due to reciprocating movement of the piston and with the mean or DC part removed. This is a smooth and unavoidable source of low frequency of about $20 \mathrm{~Hz}$, which is less harmful and hence of less interest.

Further deflation and BLMS extraction recovered the third source $\hat{\mathbf{s}}_{3}$ which is a high frequency one. It is located at about the same location as the piston slap and is believed to be the pressure oscillation component due to combustion or combustion knocks which is harmful and hence should be an interested source. In this academic study we have measured cylinder pressure hence a high pass filtered cylinder pressure is plotted in row 4 of the figure to compare with blind recovered source. Although they are not the same yet they are similar. The difference between them is that the section that has significant values for $\hat{\mathbf{s}}_{3}$ is shorter than $\mathrm{dP}$. This is due to that the inverse filtering or deconvolution of the blind algorithm drives the recovered source towards maximum super nongaussianity, i.e. there is an undetermined filtering effect. This effect could have been aggravated by the nonstationarity of the engine source, especially for the combustion pressure source not being independently identically distributed (i.i.d) but having a correlated oscillating character instead. We will not describe the i.i.d. requirement for sources in detail, but just refer to ...[7?], and also refer the paper by Antoni which describes the various characters of mechanical sources [8]. Even so, in practical engineering situation where the cylinder pressure is not available, the blind recovered source can be an approximate for evaluating the combustion performance.

To further verify the method and the separation results, the same separation process is applied to the faulty engine at a different operating speed of about $1000 \mathrm{rpm}$. Four measured acceleration mixtures are plotted in Fig. 7. Similar separation results are obtained and shown in Fig. 8 demonstrating that the combined separation method of the BLMS and the deflation algorithms can offer reliable separation results. 

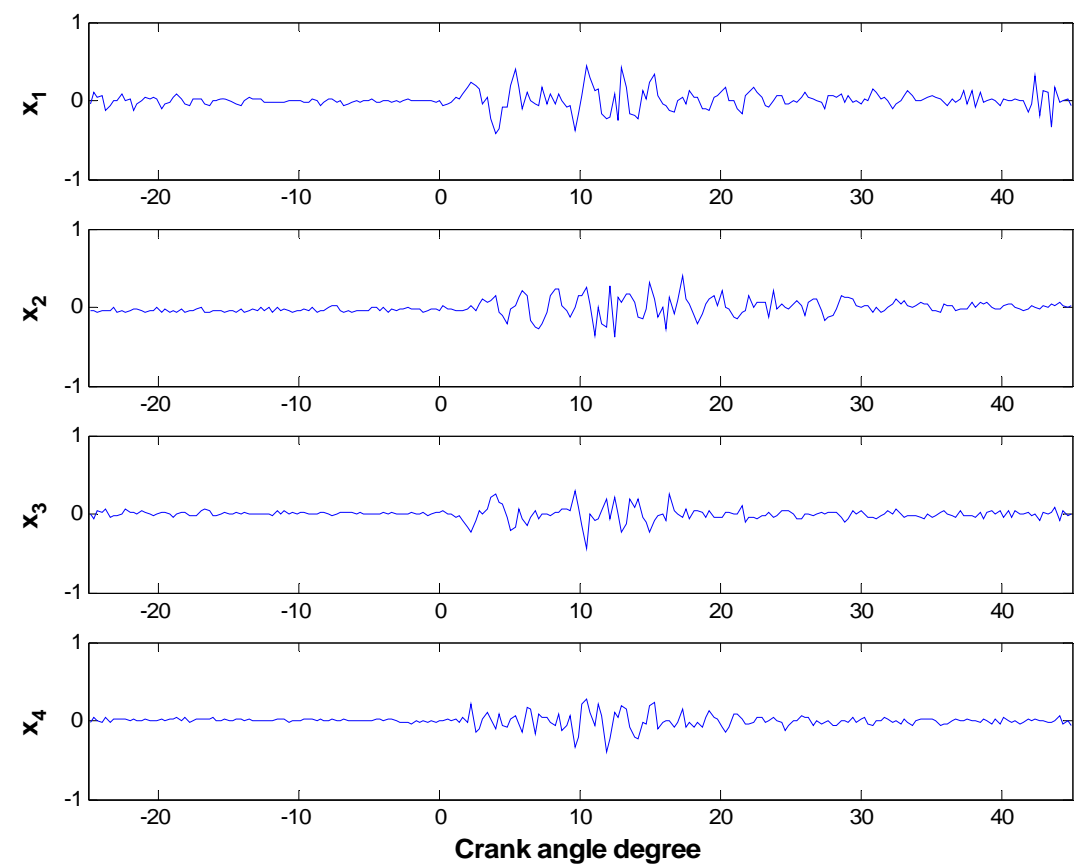

Fig. 5. Four measured vibration acceleration signals of a faulty engine running at $1210 \mathrm{rpm}$
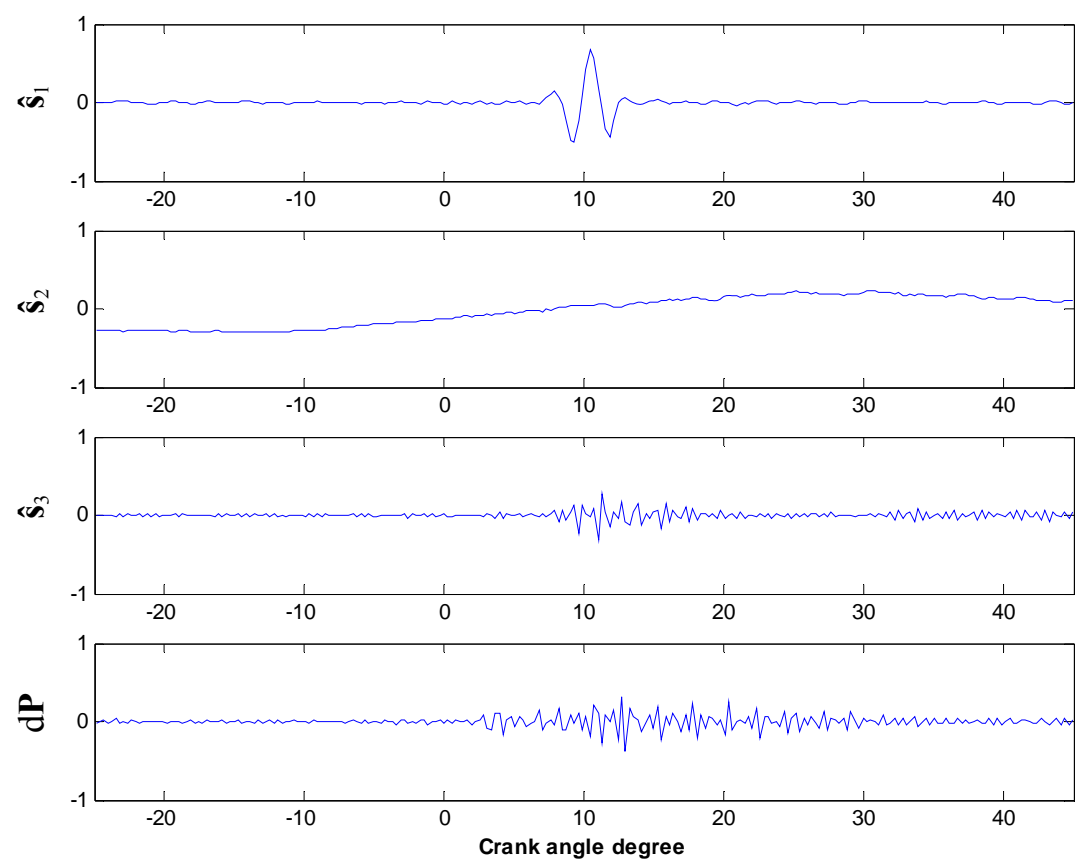

Fig. 6. Three separated sources and high pass filtered cylinder pressure (enlarged 3 times) 

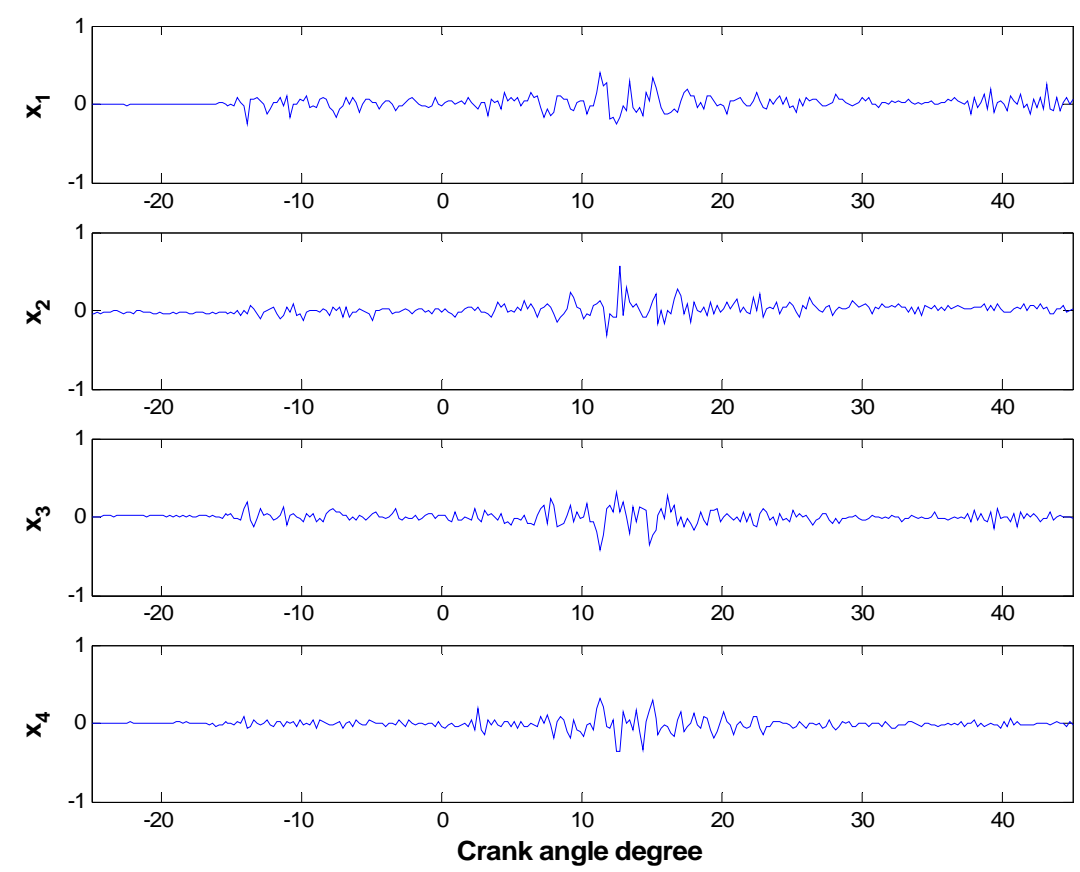

Fig. 7. Four measured vibration acceleration signals of a faulty engine running at $1000 \mathrm{rpm}$
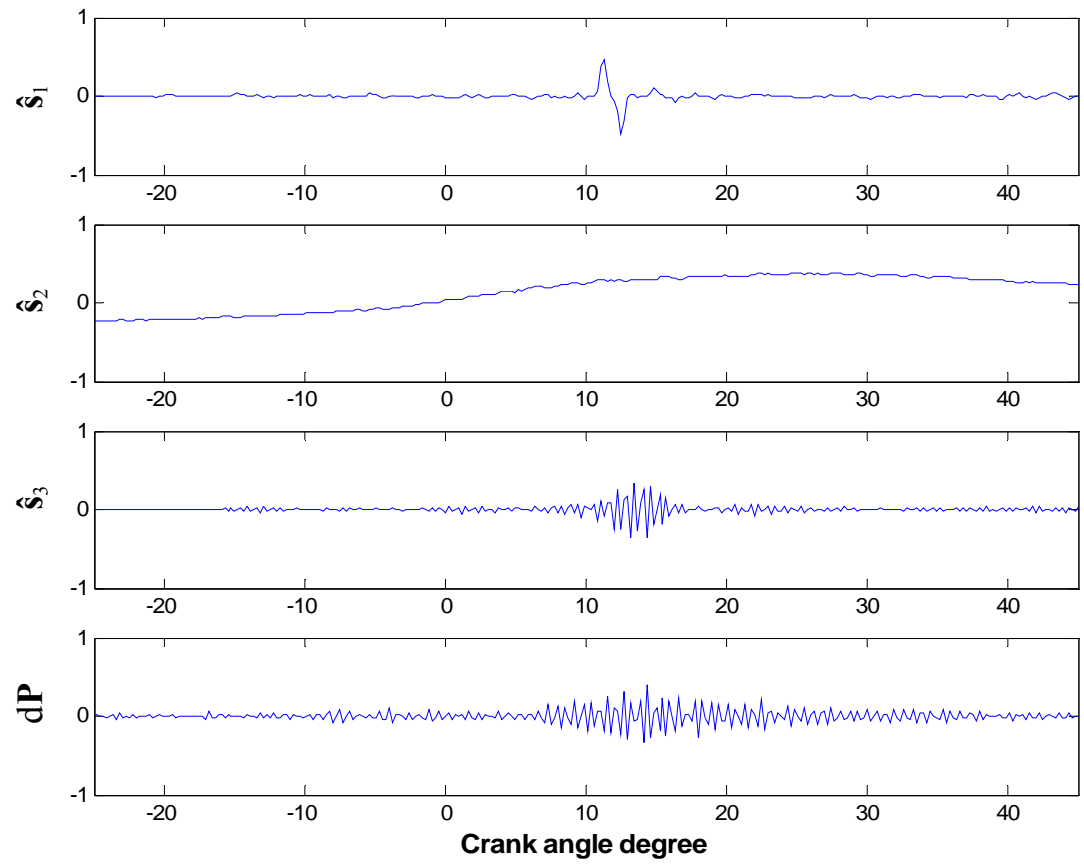

Fig. 8. Three separated sources and high pass filtered cylinder pressure (enlarged 3 times)

In summary, taking into account the success for separation of simulated sources, it is believed that the combined method has to some extent been reasonably applied to the engine vibration problem for separating more sources and has obtained reasonable results. 


\section{Conclusion and discussion}

This paper has briefly introduced the BLMS algorithm and proposed combining a deflation method for separating more sources. Separation experiment of four simulated signals of both sub and supergaussian distribution shows that the combined method is quite successful. The separation experiments of vibration signals of a faulty piston engine have obtained three sources which are reasonably interpreted as piston slap, cylinder pressure change due to the reciprocating movement of piston, and cylinder pressure oscillation due to combustion.

A comparison between the blind recovered cylinder pressure oscillation source and high pass filtered measured pressure show that the blind algorithm have a tendency to over estimate the sources towards nongaussianity. While this is partly caused due to the nonstationary non i.i.d. nature of the engine sources, the combined algorithm could be improved if some proper constraint be added which is one of our interests for future investigation. The other future investigation interest is how to utilize the blind recovered sources, for example, how to evaluate the piston performance by the recovered piston slap signal and how to evaluate the combustion performance by the blind recovered pressure oscillation so that to enable better engineering design.

\section{Acknowledgments}

The authors are grateful to the Australian Research Council for funding this Discovery project and to DSTO for support under the Centre of Expertise scheme.

\section{References}

[1] J. Antoni and S. Braun edit, Special Issue: Blind Source Separation, Mechanical Systems and Signal Processing 19 (6) (2005), Elsevier Ltd.

[2] X. Liu and R. B. Randall, Blind source separation of internal combustion engine piston slap from other measured vibration signals. Mechanical Systems and Signal Processing 19 (6) (2005), pages 1196-1128.

[3] S. Haykin edits, "Unsupervised Adaptive Filtering Volume I: Blind Source Separation", New York, Chichester, John Wiley and Sons, Inc. 2000.

[4] A. Cichocki and S. Amari, "Adaptive Blind Signal and Image Processing”, John Wiley \& Sons, LTD, 2003.

[5] S.Choi and A. Cichocki, On-line sequential multichannel blind deconvolution: A deflation approach. In Proc. $8^{\text {th }}$ IEEE DSP Workshop, pages 159-162, Utahi, USA, 1998.

[6] A. Hyvärinen, J. Karhinen and E. Oja, Independent Component Analysis, New York, Chichester, John Wiley \& Sons, 2001.

[7] Which paper is better on i.i.d. requirements?

[8] J. Antoni, Blind separation of vibration components: Principles and demonstrations, Mechanical Systems and Signal Processing 19 (6) (2005). 